\title{
Variable Impedance Actuators: Moving the Robots of Tomorrow
}

\author{
B. Vanderborght ${ }^{6}$, A. Albu-Schaeffer ${ }^{1}$, A. Bicchi ${ }^{2,5}$, E. Burdet ${ }^{4}$, D. Caldwell ${ }^{5}$, R. Carloni ${ }^{3}$, M. Catalano ${ }^{2,5}$, \\ G. Ganesh ${ }^{4}$, M. Garabini ${ }^{2}$, M. Grebenstein ${ }^{1}$, G. Grioli ${ }^{2}$, S. Haddadin ${ }^{1}$, A. Jafari ${ }^{5}$, M. Laffranchi ${ }^{5}$, D. Lefeber ${ }^{6}$, \\ F. Petit ${ }^{1}$, S. Stramigioli ${ }^{3}$, N. Tsagarakis ${ }^{5}$, M. Van Damme ${ }^{6}$, R. Van Ham ${ }^{6}$, L.C. Visser ${ }^{3}$, S. Wolf ${ }^{1}$
}

\section{INTRODUCTION}

While plants do not possess a brain to move voluntarily, animals use their brain to move at will, see, hear, taste and touch. Recently, an increasing number of researchers have realized that in animals not only the brain creates the intelligence of the body, but that the morphology and biomechanics have a great impact on the way animals and humans think and move [1]. A critical role in this respect is played by the neuromechanics of muscles, which have functional performance and control capabilities far in excess reached by artificial actuators. In traditional robotics, stiff actuators controlled as servomotor governed by the principle of "the stiffer the better", produce high bandwidth control adapted to tasks requiring tracking of a desired trajectory with high accuracy. However, the actuator's mechanics, through its own mass, inertia, stiffness etc., heavily influence the control of the whole robotic system.

Many -mostly novel- applications requiring interaction with an unknown and dynamic environment including humans require dynamics that are not well suited to servomotors. Therefore, Variable Impedance Actuators (VIA) are being developed, which are inspired by biological motor control, where the influence of muscle spring-like properties and their control are of great importance [2]. In parallel, the planning and control algorithms for using these novel actuators properties is increasingly studied. The EU-project VIACTORS addressed the development of such safe, energyefficient and highly dynamic variable impedance actuation systems, which will permit the embodiment of natural characteristics, found in biological systems into a new generation of mechatronic systems. The goal of the project is to obtain the intended physical interaction and motion behaviors of the robotic system intrinsically by its physical structures to the maximum extent possible. This advance in technology will pave the way towards new application fields, such as industrial co-workers, household robots, advanced prostheses and rehabilitation devices and other autonomous robots.

\section{VARIABLE IMPEDANCE ACTUATORS}

The idea of extending human-like impedance control [3] to robots started with the work of Hogan [4], and with earlier VIA such as McKibben pneumatic actuators [5] and

\footnotetext{
${ }^{1}$ DLR/Institute of Robotics and Mechatronics, Germany, ${ }^{2}$ University of Pisa, Italy, ${ }^{3}$ University of Twente, Netherlands, ${ }^{4}$ Imperial College London, United Kingdom, ${ }^{5}$ Istituto Italiano di Tecnologia, Italy, ${ }^{6}$ Vrije Universiteit Brussel, Belgium, http://www.viactors.eu corresponding author bram. vanderborght @ vub.ac.be
}

the series elastic actuator (SEA) [6]. Currently different principles exist to implement (variable) impedance in an actuator design. Within VIACTORS many actuators have been developed to explore their possibilities. In [7] an energy efficient actuator is achieved by a combination of an Infinite Variable Transmission and an elastic element. In AwAS [8], AwAS-II [9], vsaUT [10] and vsaUT-II [11] systems were developed where the adaptation of stiffness is achieved with little energy consumption. In the Maccepa [12] and Maccepa 2.0 [13] an easy to construct VIA was obtained, which can be constructed with off-the-shelf components. The VS-Joint [14] is the analogy of the Maccepa actuator, but using a compression spring instead with extension spring. The Floating Spring Joint [15] has been designed to use the spring energy optimally. To have the sum of the two motor torques available, bi-directional antagonistic arrangements were made [16][17]. The VSA-cube is a hobby servomotor style of VIA actuator [18] and is currently commercialised so that as wide public audience can explore the possibilities of VIAs. The Pleated Pneumatic Artificial Muscles are in an antagonistic setup adaptably compliant and can generate higher forces at lower pressures compared to McKibben muscles [19]. To facilitate the control of compliant robotic joints, actuators providing physical damping have been developed as well as a friction-based [20] and fluid dynamics-based [21] damper.

\section{APPLICATIONS}

Applications of the Variable Impedance Actuators are in tasks where the robot needs to move in physical interaction with an unknown and dynamic environment and the controlled body-actuator system must achieve abilities like:

Safety to humans (and resilience to self-damage) in operations where the robot is required to have positional accuracy and swiftness of motion, while cooperating, physically interacting or even possibly colliding with the humans and their environment, such as e.g. in collaborative robotics. This has been studied using a pneumatic arm [22] and the DLR Hand/Arm system [23], and illustrated through impacts' response (e.g. hammering on the fingers and striking using a baseball on the arm). The social robot Probo uses SEAs to produce a safe and huggable HRI with children [24].

Efficiency (e.g. natural gait generation, adaptation in legged locomotion applications and prosthetics for lower limbs), e.g. in the bipeds Lucy [25], Veronica [26], Dribbel [27] and Tulip where the natural dynamics are exploited to reduce the energy efficiency. Or the AMPfoot prosthesis were 
energy is stored and released during walking [28]. By storing energy in a spring a ball can be thrown or kicked much further than when a stiff actuator is used [14].

Robustness to external perturbations and unpredictable model errors (changes) of the environment, of the robot kinematics and dynamics, or of the dynamics of a human interacting with it. This is often required in tasks like hammering, holding cups, drumming [18]; typical tasks include the use of tools such as screwdriving, cutting, polishing [29], drawing [23] or stabilizing a humanoid [30].

Adaptability and force accuracy in the interaction with the operator, in applications in which continuous contact and accurate force exchange is necessary, such as in "hands-on" assistive devices, rehabilitation, exoskeletons and haptics. This is achieved e.g. in the Knexo knee exoskeleton [31].

\section{CONCLUSION}

Most of today's robots have rigid structures and actuators requiring complex software control algorithms and sophisticated sensor systems in order to behave in a compliant and safe way adapted to contact with unknown environments and humans. By studying and constructing variable impedance actuators and their control, we contribute to the development of actuation units which can match the intrinsic safety, motion performance and energy efficiency of biological systems and in particular the human. As such, this may lead to a new generation of robots that can co-exist and co-operate with people and get closer to the human manipulation and locomotion performance than is possible with current robots.

\section{ACKNOWLEDGMENT}

Part of the work has been funded by the European Commissions FP7 project VIACTORS under grant no. 231554.

\section{REFERENCES}

[1] R. Pfeifer, J. Bongard, and S. Grand, How the body shapes the way we think: a new view of intelligence. The MIT Press, 2007.

[2] R. M. Alexander, Exploring Biomechanics: Animals in Motion. Scientific American Library, 1992.

[3] E. Burdet, R. Osu, D. Franklin, T. Milner, and M. Kawato, "The cns skillfully stabilizes unstable dynamics by learning optimal impedance," Nature, vol. 414, pp. 446-449, 2001.

[4] N. Hogan, "Impedance control: An approach to manipulation: Part illapplications," Journal of dynamic systems, measurement, and control, vol. 107, no. 2, p. 17, 1985.

[5] D. G. Caldwell, G. Medrano-Cerda, and M. Goodwin, "Control of pneumatic muscle actuators," IEEE Control Systems Magazine, vol. 15, no. 1, pp. 40-48, February 1995.

[6] G. A. Pratt and M. M. Williamson, "Series elastic actuators," in IROS 199, Pittsburg, USA, 1995, pp. 399-406.

[7] S. Stramigioli, G. van Oort, and E. Dertien, "A concept for a new energy efficient actuator," in AIM 2008. IEEE, 2008, pp. 671-675.

[8] A. Jafari, N. Tsagarakis, B. Vanderborght, and D. G. Caldwell, "A novel actuator with adjustable stiffness (AwAS)," in IROS 2010. IEEE, 2010, pp. 4201-4206.

[9] A. Jafari, N. Tsagarakis, and D. G. Caldwell, "AwAS-II: A new actuator with adjustable stiffness based on the novel principle of adaptable pivot point and variable lever ratio," in ICRA 2011. IEEE, 2011, pp. 4638-4643.

[10] L. C. Visser, R. Carloni, and S. Stramigioli, "Energy efficient variable stiffness actuators," IEEE Transactions on Robotics, vol. 27, no. 5, pp. 865-875, 2011.

[11] S. Groothuis, G. Rusticelli, A. Zucchelli, S. Stramigioli, and R. Carloni, "The vsaUT-II: a Novel Rotational Variable Stiffness Actuator," in ICRA, 2012.
[12] R. Van Ham, B. Vanderborght, M. Van Damme, B. Verrelst, and D. Lefeber, "MACCEPA, the Mechanically Adjustable Compliance and Controllable Equilibrium Position Actuator: Design and Implementation in a Biped Robot," Robotics and Autonomous Systems, vol. 55, no. 10, pp. 761-768, October 2007.

[13] B. Vanderborght, N. Tsagarakis, R. Van Ham, I. Thorson, and D. Caldwell, "Maccepa 2.0: compliant actuator used for energy efficient hopping robot chobino1d," Autonomous Robots, pp. 1-11, 2009.

[14] S. Wolf and G. Hirzinger, "A new variable stiffness design: Matching requirements of the next robot generation," in ICRA 2008, May 2008, pp. 1741-1746.

[15] S. Wolf, O. Eiberger, and G. Hirzinger, "The DLR FSJ: Energy based design of a variable stiffness joints," in ICRA), 2011. IEEE, 2011, pp. 5082-5089.

[16] R. Schiavi, G. Grioli, S. Sen, and A. Bicchi, "VSA-II: A novel prototype of variable stiffness actuator for safe and performing robots interacting with humans," in IEEE Int. Conf. on Robotics and Automation (ICRA 2008). IEEE, 2008, pp. 2171-2176.

[17] F. Petit, M. Chalon, W. Friedl, M. Grebenstein, A. Albu-Schaeffer, and G. Hirzinger, "Bidirectional antagonistic variable stiffness actuation: Analysis, design amp; implementation," in Proc. IEEE International Conf. on Robotics and Automation (ICRA 2010), 2010, pp. 4189-4196.

[18] M. Catalano, G. Grioli, M. Garabini, F. Bonomo, M. Mancini, N. Tsagarakis, and A. Bicchi, "Vsa-cubebot: a modular variable stiffness platform for multiple degrees of freedom robots."

[19] D. C. Villegas, M. Van Damme, B. Vanderborght, and L. Dirk, "Third generation pleated pneumatic artificial muscles for robotic applications: Development and comparison with mckibben muscle," Advanced Robotics, 2012.

[20] M. Laffranchi, N. Tsagarakis, and D. Caldwell, "A variable physical damping actuator (VPDA) for compliant robotic joints," ICRA, pp. 1668-1674, 2010.

[21] M. Catalano, G. Grioli, M. Garabini, F. W. Belo, A. di Basco, N. Tsagarakis, and A. Bicchi, "Implementing a variable impedance actuator," in ICRA 2012, 2012.

[22] M. Van Damme, B. Vanderborght, B. Verrelst, R. Van Ham, F. Daerden, and D. Lefeber, "Proxy-based sliding mode control of a planar pneumatic manipulator," International Journal of Robotics Research, vol. 28, no. 2, pp. 266-284, 2009.

[23] M. Grebenstein, A. Albu-Schäffer, T. Bahls, M. Chalon, O. Eiberger, W. Friedl, R. Gruber, U. Hagn, R. Haslinger, H. Höppner, et al., "The dlr hand arm system," vol. 11, 2010.

[24] K. Goris, J. Saldien, B. Vanderborght, and D. Lefeber, "Mechanical design of the huggable robot probo," International Journal of Humanoid Robotics, vol. 8, no. 3, pp. 481-511, 2011.

[25] B. Vanderborght, R. Van Ham, B. Verrelst, M. Van Damme, and D. Lefeber, "Overview of the lucy project: Dynamic stabilization of a biped powered by pneumatic artificial muscles," Advanced Robotics, vol. 22 , no. 25, pp. 1027-1051, 2008.

[26] R. Van Ham, B. Vanderborght, M. Van Damme, B. Verrelst, and D. Lefeber, "MACCEPA, the mechanically adjustable compliance and controllable equilibrium position actuator: Design and implementation in a biped robot," Robotics and Autonomous Systems, vol. 55, no. 10, pp. 761-768, October 2007.

[27] E. Dertien, "Dynamic walking with dribbel," IEEE Robotics \& Automation Magazine, vol. 13, no. 3, pp. 118-122, September 2006.

[28] P. Cherelle, A. Matthys, V. Grosu, and D. L. Bram Vanderborght, "The amp-foot 2.0: Mimicking intact ankle behavior with a powered transtibial prosthesis." in BioRob2012, 2012.

[29] C. Yang, G. Ganesh, S. Haddadin, S. Parusel, A. Albu-Schaeffer, and E. Burdet, "Human-like adaptation of force and impedance in stable and unstable interactions," Robotics, IEEE Transactions on, vol. 27, no. 5, pp. 918-930, 2011.

[30] L. Zhibin, B. Vanderborght, N. Tsagarakis, L. Colasanto, and D. G. Caldwell, "Stabilization for the compliant humanoid robot coman exploiting intrinsic and controlled compliance," in IEEE-RAS ICRA, 2012, pp. $2000-2006$.

[31] P. Beyl, M. Van Damme, R. Van Ham, B. Vanderborght, and D. Lefeber, "Design and control of a lower limb exoskeleton for robotassisted gait training," Applied Bionics and Biomechanics, vol. 6, no. 2, pp. 229-243, 2009. 